\title{
Dexmedetomidine Reduces IL-4 and IgE Expression Through Downregulation of TLR4/NF-K Signaling Pathway to Alleviate Airway hyperresponsiveness in OVA Mice
}

Qirui Duan ( $\sim 592901066 @ q q . c o m$ )

Plastic surgery Hospital https://orcid.org/0000-0002-5286-3145

Dong Yang

Plastic Surgery hospital

Juan Zhi

plastic surgery hospital

\section{Research Article}

Keywords: Dexmedetomidine, Airway Hyperresponsiveness, Asthma, TLR4/NF-KB, IL-4

Posted Date: September 28th, 2021

DOl: https://doi.org/10.21203/rs.3.rs-939761/v1

License: (c) (i) This work is licensed under a Creative Commons Attribution 4.0 International License.

Read Full License 


\section{Abstract}

Background: Asthma is a disease that affects health worldwide. It is characterised by inflammation and airway hyperreactivity. Because airway hyperreactivity can occur in other diseases, perioperative airway hyperreactivity is more insidious and widespread than in asthma and has serious implications that need to be addressed urgently. The use of dexmedetomidine in acute asthma and lung protection has been reported, but the exact mechanism is unclear.

Objective: To investigate the effectiveness and mechanisms associated with dexmedetomidine in airway hyperresponsiveness.

Methods: Forty BALB/c female mice were randomly divided into five groups: group K (blank group), group A (asthma group), group HD (asthma + dexmedetomidine treatment group), group TH (asthma + yohimbine group) and group HT (asthma + dexmedetomidine + yohimbine group), and the airway resistance of group $\mathrm{K}$, group $\mathrm{A}$ and group HD were analysed by invasive airway resistance assay, ELISA assay, immunohistochemistry and q-PCR, respectively. Airway resistance; IL-4 and IgE levels in serum and BLAF; and IL-4, IL-13, Muc5AC, NFKB, TLR2, TLR4 and TSLP1 protein levels in lung tissues of the 5 groups were analysed by invasive airway resistance assay, ELISA, immunohistochemistry and qPCR.

RESULTS: Compared with group A, there were statistical differences in airway resistance $(\boldsymbol{P}<0.05)$; LIL-4 and IgE $(\boldsymbol{P}<0.05)$ in serum and BLAF; and Muc5AC, TLR4 and NFKB protein contents $(\boldsymbol{P}<0.05)$ in lung tissues in the HD group.

Conclusion: 1. Dexmedetomidine can attenuate airway hyperresponsiveness in the OVA asthma model; 2. Dexmedetomidine reduced the production of IL-4 and IgE by down-regulating the TLR4/NF-KB signaling pathway, thereby reducing the lung inflammatory response and airway hyperresponsiveness in the OVAinduced asthma model.

\section{Introduction}

Asthma is a heterogeneous respiratory disease that is estimated to affect over 300 million people worldwide, with up to $10 \%$ of adults and $30 \%$ of children suffering from asthma ${ }^{[1]}$, and causes 380,000 deaths each year ${ }^{[2]}$. The Lancet reported in 2015 that the prevalence of asthma in China was $4.2 \%{ }^{[3]}$. According to the Global Initiative for Asthma 2020 (GINA), the prevalence of asthma is still rising in many countries ${ }^{[4]}$. It is now generally accepted that airway hyperreactivity is the main pathological feature and diagnostic indicator of airway asthma. Airway Hyper-Responsiveness (AHR) is caused by chemical or physical factors (internal or external) that can trigger excessive and premature contraction of airway smooth muscle and increased mucus secretion and microvascular leakage ${ }^{[5]}$. In addition, many clinical diseases (including asthma, chronic obstructive pulmonary disease, allergic euthyroidism, novel coronavirus pneumonia, gastro-oesophageal reflux, etc.) and environmental (irritants, PM2.5, etc.) deterioration can cause neurosensitization, airway inflammation, etc. and thus airway hyperreactivity, 
while some clinical patients with underlying allergies, unknowingly using some anesthetic drugs or fluids can cause increased airway pressure. Airway hyperreactivity is a clinical manifestation that is insidious and unpredictable. In the perioperative period, when triggered by specific factors, there is a tendency for airway spasm, sputum (and airway narrowing) to cause mechanical ventilation difficulties leading to hypoxaemia and hypotension. In severe cases, a "silent lung" may develop, which can be life-threatening. Asthma is a serious global health problem ${ }^{[6]}$ and airway hyperresponsiveness is not only a major clinical manifestation of asthma, but also of other diseases, making it more widespread than asthma. In general, preoperative use of $\beta 2$ agonists can be used as treatment or prevention for asthma patients, but there are no good preventive measures for patients with other potential airway hyperresponsiveness in the perioperative period, especially during peri-anaesthesia. In order to effectively protect patients' total airway safety and life safety during the perioperative period, it is an important issue to identify the mechanism of airway hyperreactivity and to provide effective preventive and therapeutic measures.

Dexmedetomidine is a highly selective a2-adrenergic agonist with sedative, analgesic and anxiolytic effects, and is one of the most widely used drugs in clinical anesthesia. The protective effect of dexmedetomidine on lung injury has been studied extensively ${ }^{[7,8]}$. In addition, the immunomodulatory effects of dexmedetomidine have been noted ${ }^{[9]}$. Studies have shown that dexmedetomidine affects immunity by modulating specific Th2 helper cells ${ }^{[10,11]}$, which in turn are major players in asthma, mediating inflammatory responses and airway hyperreactivity through the production of type 2 cytokines (IL-4, IL-13, thymic stromal lymphopoietin (TSLP), etc.) ${ }^{[11]}$; dexmedetomidine reduces inflammatory factor secretion by attenuating NFKB signalling pathway phosphorylation in monocytes and macrophages ${ }^{[12]}$. Meanwhile Hamida Hammad [11] noted that most allergens and many air pollutants trigger the NFKB signalling pathway to promote Th2 development by activating toll-like receptors (TLRs), among others, activating innate immune cells and subsequently producing many mediators that contribute to airway inflammation.Martijn L. Manson ${ }^{[13]}$ found that it was IL-13 and IL-4 We therefore hypothesise that dexmedetomidine reduces IL4/13 by downregulating the TLR4/NF-KB signalling pathway to alleviate airway hyperresponsiveness in OVA mice, thus achieving prevention of perioperative airway hyperresponsiveness.

\section{Materials And Methods}

Forty female BALB/c mice, 5-7 weeks old, were purchased from the Experimental Animal Centre of the Hospital of Orthopaedic Surgery, Chinese Academy of Medical Sciences and housed in the SPF Animal Centre of the Hospital of Orthopaedic Surgery, Chinese Academy of Medical Sciences. The mice were fed with sterilized chow and drinking water, and after one week of acclimatization in the animal house, a mouse airway hyperresponsiveness model was started. They were also treated and housed in groups according to the different interventions.

\subsection{OVA sensitisation protocol and dexmedetomidine treatment}


2.1.1 Asthma model: BALB/c mice, sensitised by intraperitoneal injection of ovalbumin (OVA) sensitising solution, were nebulised using a PRONEB compression nebuliser during the excitation period, and the model was successfully established for a total of 55 days.

\subsubsection{Evaluation of successful asthma model establishment}

Airway responsiveness monitoring: $48 \mathrm{~h}$ after the last nebulised excitation, the airway responsiveness of $\mathrm{BALB} / \mathrm{c}$ mice was measured using an American invasive small animal lung function meter. Different concentrations of Methacholine (Mch $₫$ Sigma) were nebulised and the Enhanced Pause (Penh) values were measured at the corresponding concentrations. The Penh value at normal saline (NS) inhalation was used as the base value and the airway responsiveness was evaluated using the maximum Penh/NS\% and PC100 and compared with the control group.

\subsubsection{Dexmedetomidine treatment protocol and grouping}

After successful establishment of asthma, the mice were divided into 3 groups: Asthma group (A), Dexmedetomidine(Jiangsu Hengrui Pharmaceutical Co.)group(HDgroup), Yohimbine (Sigma)group(TH group) and Dexmedetomidine + Yohimbine group (HT group); group A was given the same dose of saline; group HD, a single intraperitoneal injection of dexmedetomidine $25 \mathrm{ug} / \mathrm{kg}$ for 5 days; group $\mathrm{TH}$, an intraperitoneal injection of yohimbine $1 \mathrm{mg} / \mathrm{kg}$ for 5 days; in the HT group, a single intraperitoneal dose of dexmedetomidine $25 \mathrm{ug} / \mathrm{kg}$ + yohimbine $1 \mathrm{mg} / \mathrm{kg}$ for 5 days.

\subsection{Access to materials}

\subsubsection{Collection of peripheral blood}

Peripheral blood was collected within 24 hours of AHR stimulation. Mice were anaesthetised with Sevoflurane (Baxter Healthcare Corporation) per mouse and blood was collected from the tail vein after anaesthesia. 50-100ul of blood was collected and left at room temperature for 1 to 2 hours, centrifuged at $3000 \mathrm{r} / \mathrm{min}$ for 10 minutes and the serum was separated and stored at $-20^{\circ} \mathrm{C}$ for ELISA testing.

\subsubsection{Bronchoalveolar lavage fluid(BALF)}

After collecting blood specimens from mice under anaesthesia, the mice were fixed and disinfected, the trachea was surgically exposed, a small incision was made, a sterile $22 \mathrm{G}$ indwelling needle was inserted (fixed to avoid damaging the lung too deeply), the left lung was immediately ligated, $0.5 \mathrm{ml}$ of $0.9 \% \mathrm{NaCl}$ solution at $4{ }^{\circ} \mathrm{C}$ was slowly injected into the right lung with a syringe each time, and then slowly withdrawn. The total recovery was about $1.2 \mathrm{ml}$ (80-90\% recovery). The lysate was filtered through gauze and the resulting BALF was centrifuged at $2000 \mathrm{r} / \mathrm{min}$ for 10 minutes at $4^{\circ} \mathrm{C}$. After centrifugation, the cells were resuspended in PBS and then stained with Giemsa stain, and the total number of cells and cell sorting were counted under a light microscope; the supernatant was separated and stored at $-20^{\circ} \mathrm{Cfor}$ ELISA. 


\subsubsection{Lungs.}

After obtaining the BALF, the catheter was removed and the lung tissue was quickly freed and separated. The middle lobe of the right lung was fixed in paraffin, sectioned, and stained for $\mathrm{HE}$ and immunohistochemistry. The fresh left lung was frozen at $-80^{\circ} \mathrm{C}$ and subjected to Real-time PCR.(Lung specimens were collected within 24 hours of the end of the last nebulisation stimulation and intervention. Mice were first anaesthetised by inhalation for 1 minute in a glass cylinder of ether, then their heads and limbs were fixed to the table in a "large" position, the lungs and heart were exposed by opening the chest, the left lung was quickly ligated with a thin string, the left lung tissue was cut out and immediately packed into DEPC water-treated EP tubes and stored in a $-80^{\circ} \mathrm{C}$ freezer for PCR. The lung tissue was then intubated through the right ventricle and rinsed by rapid perfusion with saline at $4^{\circ} \mathrm{C}$ until the lung lobes turned white. Finally, the lungs were embedded in paraffin wax and sliced to a thickness of approximately $4 \mu \mathrm{m} .6$ sections of each specimen were selected for HE staining and immunohistochemical staining. Six sections of each specimen were selected for HE staining and immunohistochemical staining.

\subsection{Experimental steps}

\subsubsection{HE stain}

HE stain steps in sequence: section;bak;dewa;gradient alcohol hydration;hematoxylin stain;ethanol acidification with hydrochloric acid;staining with eosin solution;Conventional dehydration, transparency: follow step (4) in reverse, same time;seal the section;Observe the airway pathological changes in each group of mice under a high magnification field of light microscopy $(10 \times 40)$.

Inflammation was scored by observing the HE-stained lung changes in each group of mice under a light microscope using a high-powered field of view (40x), and was divided into 0-4 points according to the degree of inflammatory cell infiltration around the bronchioles: 0 , normal bronchioles with no inflammatory cell infiltration; 1 , bronchioles with less than 1 layer of inflammatory cell infiltration; 2 , bronchioles with 1 layer of inflammatory cell infiltration; 3 , bronchioles with 2-4 layers of inflammatory cell infiltration; 4 , bronchioles with more than 4 layers of inflammatory cell infiltration. infiltrates fine bronchi; 4 points, more than 4 layers of inflammatory cells infiltrate fine bronchi.

2.3.2 Immunohistochemistry: observe MUC5AC, TLR4 and NF-KB expression, following the instructions in sequence. (Rabbit anti-MUC5AC,anti-TLR4,anti-NF-KB polyclonal antibody Biyuntian Institute of Biotechnology).

\subsubsection{AB-PAS staining}

PAS (Periodic Acid-Schif0 staining kit purchased from Sigma, product no. 395B. AB (Alcian Blue 8GX) purchased from Sigma. product no. A3157. pH 2.5 in AB8GX solution: 1\% AB 8GX dissolved in 3\% acetic acid. 
The left lung of the mice was soaked in $4 \%$ neutral formaldehyde, and the soaking solution was replaced with $30 \%$ sucrose water after $12 \mathrm{~h}-24 \mathrm{~h}$ of soaking, and the lung tissue continued to be soaked at room temperature for $12 \mathrm{~h}-24 \mathrm{~h}$. After staining, the peribronchial cupped cell hyperplasia was observed microscopically and scored: the bronchi of each group of mice were observed under a high magnification light microscope (10×40), and the degree of cupped cell hyperplasia was mainly evaluated microscopically. The score of cupped cell hyperplasia was 0-4 according to the proportion of cupped cells in the peritubular area of the bronchial wall: 0 , no cupped cells in the bronchial epithelium; 1 , less than $25 \%$ of the peritubular area of the bronchial wall; $2,25 \%-50 \%$ of the peritubular area of the bronchial wall; $3,51 \%-75 \%$ of the peritubular area of the bronchial wall; 4 , cupped cells in the peritubular area of the bronchial wall The proportion of cup-shaped cells in the peritubular area of the bronchial wall is $>75 \%$.

\subsubsection{ELISA}

The mouse serum and BALF supernatant stored at $-20^{\circ} \mathrm{C}$ were removed and the IL- 4 and IgE levels in the BALF fluid and peripheral blood were measured in strict accordance with the ELISA kit instructions. (Mouse IgE Elisa Kit Dr.D.

\subsubsection{Real-time PCR}

RNA Extraction; Reverse transcription; PCR Detection; Primer Sequence;

\section{The following table-1 provides information on primers}




\begin{tabular}{|c|c|c|c|c|}
\hline Gene Name & Primer name & Primer(5-3) & Amplicon length(bp) & Gene ID \\
\hline \multirow[t]{2}{*}{ TLR2 } & Ms-TLR2-F & GCAAACGCTGTTCTGCTCAG & 231 & 24088 \\
\hline & Ms-TLR2-R & AGGCGTCTCCCTCTATTGTATT & & \\
\hline \multirow[t]{2}{*}{ TLR4 } & Ms-TLR4-F & ATGGCATGGCTTACACCACC & 129 & 21898 \\
\hline & Ms-TLR4-R & GAGGCCAATTTTGTCTCCACA & & \\
\hline \multirow[t]{2}{*}{ IL-13 } & Ms-IL-13-F & CCTGGCTCTTGCTTGCCTT & 116 & 16163 \\
\hline & Ms-IL-13-R & GGTCTTGTGTGATGTTGCTAC & & \\
\hline \multirow[t]{2}{*}{ IL-4 } & Ms-IL-4-F & GGTCTCAACCCCCAGCTAGT & 102 & 16189 \\
\hline & Ms-IL-4-R & GCCGATGATCTCTCTCAAGTGAT & & \\
\hline \multirow[t]{2}{*}{ TSLP } & Ms-TSLP-F1 & AGAAGCCCTCAATGACCAC & 104 & 53603 \\
\hline & Ms-TSLP-R1 & AGGTTTGATTCAGGCAGAT & & \\
\hline \multirow[t]{2}{*}{ Muc5AC } & Ms-Muc5AC-F1 & GTGGTTTGACACTGACTTCCC & 103 & 17833 \\
\hline & Ms-Muc5AC-1 & СTCCTCTCGGTGACAGAGTCT & & \\
\hline \multirow[t]{2}{*}{ NF-kb p65 } & NF-kb p65-F1 & AGGCTTCTGGGCCTTATGTG & 111 & 19697 \\
\hline & NF-kb p65-R1 & TGCTTCTCTCGCCAGGAATAC & & \\
\hline
\end{tabular}

PCR amplification was carried out according to the DBI Bestar® SybrGreen q-PCR masterMix instructions, and the reaction system for Real time PCR amplification was $20 \mu \mathrm{l}$.

\section{Real time PCR reaction system}

Determination of Real-time PCR results

At the end of the experiment, the Ct value of each PCR measurement was recorded and averaged three times, using $\beta$-action as the internal reference. The data were processed according to the $2-\triangle \triangle \mathrm{Ct}$ method. $\triangle \mathrm{Ct}=$ mean \pm standard deviation of $\mathrm{Ct}$ values for (target gene - reference gene); $\triangle \triangle \mathrm{Ct}=\mathrm{mean}$ \pm standard deviation of $\triangle \mathrm{Ct}$ for (sample to be tested - reference sample); mean \pm standard deviation of initial template amount for relative samples $=(2-\triangle \triangle \mathrm{Ct})$. Relative expression of target gene $(\mathrm{RQ}$ value $)=$ $2-\triangle \triangle \mathrm{Ct}$ 


\subsection{Statistical analysis}

In this study, Imege $\mathrm{J}$ was used for image processing. The data were analysed using GraphPad Prism 8.0 software. One-way ANOVA was used for comparison when the means of each group met the chi-square; when the means of each group did not meet the chi-square, the rank sum test was used. A multivariate ANOVA was used for comparison between groups. When $\mathrm{P}<0.05$, the difference is statistically significant, and when $\mathrm{P}<0.01$, the difference is statistically significant.

\section{Results}

\subsection{Increased airway inflammatory response and presence of airway hyperresponsiveness in the OVA asthmatic BALB/c female mouse model.}

After the OVA-induced allergic asthma BALB/c female mouse model was established, airway responses were stimulated by incremental doses of acetylcholine, and airway resistance was measured (as shown in Figure 3-A), which was statistically different in group A compared to group $\mathrm{K}$ at $6 \mathrm{~h}, 12 \mathrm{~h}, 24 \mathrm{~h}$ and $46 \mathrm{~h}$ after OVA induction. Further observation of pathological tissues (HE and PAS staining, found in Figure 3-B and $\mathbf{C}$ ), compared with group $\mathrm{K}$, a large number of inflammatory cells were seen in the fine bronchial wall and interstitial lung stroma in group A, and there was significant destruction of lung tissue; also visible was cupular cell hyperplasia and increased mucus secretion, which was also statistically significant after scoring analysis (Figure D). IL-4 and IgE were also measured in blood (Blood) and bronchoalveolar lavage fluid (BLAF) by ELISA (Fig3- E, F, G), and were statistically different in group A compared to group K. To later investigate the underlying mechanism, we measured Muc5Ac, NFKB, and TLR4 in lung tissue by immunohistochemistry (Figure 3-H), which were significantly higher in lung tissue in group A compared with group K (Figure I, $\boldsymbol{P}<0.001$ ). IL-4 was elevated in lung tissue, blood, and BALF fluid after OVA exposure; IgE was elevated in blood IL-4 was elevated in lung tissue, blood and BALF fluid; IgE was elevated in blood and BALF fluid; Muc5AC, NFKB and TLR4 were elevated in lung tissue.

\subsection{Dexmedetomidine attenuates airway hyperresponsiveness after OVA exposure.}

After five consecutive days of treatment with dexmedetomidine $25 \mathrm{ug} / \mathrm{kg}$ by intraperitoneal injection respectively, invasive airway resistance was measured by the same method in the HD group of OVA asthma mouse model. Compared with group A, airway resistance in the HD group was different at 48h, interestingly, airway resistance was significantly reduced at $12 \mathrm{~h}$, but $\mathrm{P}=0.05$, no statistical difference, but there was a statistical difference from 12h-48h (Figure 2-A).

\subsection{Dexmedetomidine improves lung pathology after OVA exposure}

The total number of eosinophils and neutrophils in the lungs of the HD group was found to be significantly reduced by Giemsa staining and counting of eosinophils and neutrophils under electron microscopy (Figure5-A) compared to group A. There was a statistically significant difference (Figure 5-B). Further observation of pathological tissues (HE and PAS staining, found in Figures 5-C and 5-E) showed a 
reduction in inflammatory cell infiltration in the fine bronchial walls and interstitial lung stroma and a significant reduction in lung tissue destruction in group $\mathrm{K}$ compared to group $\mathrm{A}$. After scoring the two groups by multiple hypothesis testing (Tukey HSD post hoc test (Figure 5-D), HD was lower than the mean of A. The difference between the two groups was -13.791 (- 19.704--7.877), a statistically significant difference ( $\boldsymbol{P}<0.001)$; meanwhile, reduced mucus secretion was found after PAS staining, and after scoring analysis (Tukey HSD post hoc test) (Figure 5-F), cupulocytosis was diminished and the HD group was below the mean of $A$, with a difference of -20.488 between the two groups (-27.192 - - 13.785), with a statistically significant difference $(\boldsymbol{P}<0.001)$.

\subsection{Dexmedetomidine inhibits the expression of markers of allergic airway inflammation following OVA exposure.}

In OVA-exposed mice treated with dexmedetomidine, IL-4 levels in peripheral blood and BLAF fluid were measured by ELISA and analysed by t-test, and were significantly reduced in the HD group compared to group A. The difference was $0.018(0.002-0.033),(P=0.036)$ in blood (Figure6-A) and in BLAF fluid (Figure6-B) 0.076 (0.05 - 0.102), the difference was statistically significant ( $P=0.001)$. In contrast, there was no statistical difference between the TH group (group with a2-blocker, yohimbine) and group A (Figure-6C), and the IL-4 protein variables in lung tissue were detected by qPCR (Figure 6-E), which was also statistically different. Subsequently, we detected the immunomarker IgE in blood and BALF fluid by ELISA (Figure-6D), and there was a statistical difference in both HD groups compared to group A. It can be seen that dexmedetomidine reduces the markers of allergic airway inflammation IL-4 and IgE after OVA exposure.

\subsection{Dexmedetomidine inhibits the inflammatory response through TLR4/NFKB2 channels, but not TLR2; it can affect IL-4 but not IL-13.}

To clarify the specific mechanism by which dexmedetomidine attenuates the inflammatory response after OVA exposure, it was previously reported that the TLR/NFKB channel is one of the important channels causing the inflammatory markers in the allergic lung. It is known from previous studies that TLR4/NFKB is significantly elevated after OVA exposure. Therefore, we once again examined Mu5AC, TLR4 and NFKB in the HD group and the $A$ and HT groups (dexmedetomidine + yohimbine group) by immunohistochemistry. Compared with the A and HT groups, Mu5AC, TLR4 and NFKB were significantly lower and statistically different in the HD group; compared with the A group, there was no statistical difference in the HT group. To clarify the specific changes in proteins in the A and HD groups, the protein content of IL-4, IL-13, Muc5AC, NFKB, TLR2, TLR4 and TSLP1 in lung tissue was measured by q-PCR. There were no significant changes in IL-13 and NFKB in lung tissue in the HD group compared to group $A$. It is possible that dexmedetomidine attenuated the inflammatory response independently of TLR2/NFKB and had no effect on IL-13.

\section{Discussion}


Asthma is a disease that affects health worldwide. Airway hyperresponsiveness is not only the main clinical manifestation and pathophysiological feature of asthma, but is also stimulated by certain specific substances in the environment. It can cause hypoxaemia and even asphyxia in the perioperative period. Current research suggests that the mechanisms involved in airway hyperresponsiveness are neurosensitisation, inflammatory bursts, activation of the immune response, epithelial cell chemotaxis, cupped cell proliferation and increased mucus secretion ${ }^{[11,14-16]}$.

Hamida Hammad ${ }^{[11]}$ and others published in cell journal concluded that most allergens and many air pollutants trigger epithelial cell nuclear factor kB (NF-kB) production by activating toll-like receptors (TLRs) or protease-activated receptors (PARs), leading to activation of dendritic cells (DCs) that migrate to priming nodes to promote Th2 development and activate the recruitment of innate immune cells to the airways, which subsequently produce many of the mediators that contribute to airway inflammation. Related studies have reported that neutrophils amplify recruitment and inflammatory responses in neutrophilic asthma by stimulating airway epithelial cells to activate the TLR4/NF-KB pathway and secrete chemokines ${ }^{[17]}$; improvement of lipopolysaccharide-induced injury, inflammation and MUC5AC expression in bronchial epithelial cells by inhibiting TLR4/NF-KB signalling has also been reported ${ }^{[18]}$. In contrast, MUC5AC is one of the main proteins of airway hyperresponsiveness, causing more mucous sputum secretion by cupped cells ${ }^{[19,20]}$. It can be seen that TLR4/NF-KB signalling pathway has an important role in airway inflammatory response and airway hyperresponsiveness. In our study, we found that TLR4/NF-KB was significantly increased after OVA exposure, as were the inflammatory factor IL-4 and the antigen-presenting agent IgE. This is consistent with the relevant studies reported above.

Dexmedetomidine is a highly selective $a 2$ adrenergic agonist (a2: $a 1$ selectivity of 1600:1). a2 receptors are $\mathrm{G}$ protein-coupled receptors with norepinephrine and epinephrine as ligands. By binding to ligands it leads to $\mathrm{Gi} / \mathrm{G} 0$ protein coupling, attenuates adenylyl cyclase, inhibits voltage-gated $\mathrm{Ca}^{2+}$ channels and promotes $\mathrm{K}^{+}$inward flow thereby hyperpolarising cells. a2 receptors are widely expressed in the central and peripheral nervous system as well as on other cells and tissues such as leukocytes and endothelial cells ${ }^{[9,21]}$.

Currently, dexmedetomidine is one of the most widely used anaesthetic agents in the perioperative period and in the ICU. Dexmedetomidine has been widely reported to reduce ventilator-induced lung injury by activating the ERK1/2 pathway ${ }^{[7]}$ and to reduce non-ventilator-related lung injury by modulating the immune phenotype of macrophages ${ }^{\text {[2]; }}$ it can also improve endotoxin-induced acute lung injury in vivo by maintaining mitochondrial dynamic homeostasis through the HIF-1a/HO-1 signalling pathway ${ }^{[8]}$; it has also been related demonstrated its effectiveness in chronic obstructive pulmonary disease ${ }^{[23]}$. In the present study, dexmedetomidine was shown to attenuate airway resistance in an OVA-induced asthma model, and its protective effect on the lung was again confirmed by sectioning and staining of lung tissue. 
Inflammatory responses and immune responses are key to the development and progression of airway hyperresponsiveness. The main specific cytokines associated with inflammation are IL-4, IL-5 and IL-13 secreted by Th2 cells; the main protein associated with the immune response allergen-specific B cells synthesise immunoglobulin $\mathrm{E}(\mathrm{IgE}){ }^{[9,14]}$. In the current study, IL-4 and $\lg$ E were measured in each group by ELISA and dexmedetomidine was found to moderate lung inflammation by modulating IL-4 and IgE. However, analysis of IL-13 in each group by q-PCR revealed that dexmedetomidine did not have a statistically significant modulating effect on IL-13. We know that both IL-4 and IL-13 are secreted by Th2 cells, however, IL-4 production is dependent on calcium-regulated neurophosphatase, whereas IL-13 production is only partially dependent on calcium-regulated neurophosphatase ${ }^{[16]}$. Calcium-regulated neurophosphatase is positively correlated with intracellular $\mathrm{Ca}^{2+}{ }^{[24]}$, and dexmedetomidine is an a2 receptor agonist, and a2 receptor agonism inhibits voltage-gated $\mathrm{Ca}^{2+}$ channels ${ }^{\left[{ }^{[}\right]}$. Therefore, we conjecture that dexmedetomidine may reduce intracellular $\mathrm{Ca}^{2+}$ concentration by inhibiting $\mathrm{Ca}^{2+}$ inward flow, thereby reducing the amount of calcium-regulated neurophosphatase and may be partly responsible for the lower IL-4 production. Unfortunately, we did not consider that IL-4 and IL-13 are not produced in the same direction at the beginning of our project, so we did not design the neural calcium-regulated neurophosphatase blockade.

Toll-like receptors (TLRs) are a subfamily of pattern recognition receptors that recognise invading pathogens and endogenous noxious stimuli to induce innate and adaptive immune responses. Among the TLRs, TLR4 is the major lipopolysaccharide (LPS) receptor. When expressed on the cell membrane TLR4 activates downstream mediators, including the transcription factor nuclear factor (NF)-kB. TLR4/NF-kB can increase cytokines (e.g. tumour necrosis factor (TNF)-a, interleukin (IL)-1 $\beta$, IL-6), chemokines, enzymes, reactive oxygen species ${ }^{[25]}$. Several studies have demonstrated that the inflammatory response can be suppressed by inhibiting the TLR4/NF-KB signalling pathway ${ }^{[25,26]}$. In anti-inflammatory lung injury ${ }^{[27]}$ and protection against ischemia-reperfusion injury ${ }^{[28]}$, dexmedetomidine has been reported to attenuate the inflammatory response by inhibiting the TLR4/NF$\mathrm{KB}$ signalling pathway. In this study, the expression of TLR4/NF-KB in different groups was analysed by immunohistochemistry and q-PCR, which not only reaffirmed the pulmonary protective effect of dexmedetomidine, but also confirmed the anti-inflammatory effect and attenuated airway hyperreactivity in the airways. In immunohistochemistry, the effect of dexmedetomidine was attenuated in the yohimbine group, showing that $\mathrm{a} 2$ receptors are involved in the inhibition of TLR4/NF-KB signalling pathway generation. However, no effect on TLR2 was seen, and related reports suggest that nebulised lidocaine inhibits airway smooth muscle cell proliferation and migration by downregulating TLR2 to improve allergic airway inflammation ${ }^{[29]}$ and blocking the TLR2/NF-KB/NFATc1 signalling pathway ${ }^{[30]}$. According to the literature, activation and correct assembly of TLR4 with upstream differentiation protein 2 (MD-2) requires relatively stable concentrations of cations of substances such as $\mathrm{Ca}^{2+}$ and $\mathrm{Mg}^{2+}{ }^{25,31]}$, while TLR2 is upstream of Pam3CSk4 ${ }^{[29,33]}$. We speculate that perhaps dexmedetomidine affects MD2 and thus TLR4/NF-KB expression. 
Muc5AC protein is the major gel-forming mucin in airway secretions. Increased airway mucus contributes to airway obstruction in respiratory diseases such as asthma and chronic obstructive pulmonary disease. muc5AC gene and protein expression is regulated by cytokines such as IL-4 and IL-1 ${ }^{[32]}$. In this experiment, Muc5AC protein levels were measured by q-PCR in the asthma and dexmedetomidine treated groups and were found to be suppressed by dexmedetomidine in line with changes in IL-4.

Earlier, according to DS Ferreira et al ${ }^{[34]}$, thymic stromal lymphopoietin1 (TSLP1) was reported to be highly expressed in the airways of acute lethal asthma, together with TLR4. Recently, it was reported that eosinophils are elevated in patients with severe asthma and can activate lung epithelial cells to produce IL-33 and TSLP ${ }^{[35]}$. TSLP 1 is frequently expressed in severe asthma. In this study, TSLP1 expression was lower in the dexmedetomidine group than in the asthma group as determined by q-PCR. The specific intrinsic regulatory mechanisms were not addressed in this study, but only correlations were seen, which may provide new therapeutic ideas for future patients with severe or fatal asthma.

In summary, dexmedetomidine could downregulate the expression of TLR4/NF-KB signalling pathway by inhibiting $\mathrm{Ca}^{2+}$ inward flow, which in turn affected the secretion of cytokine IL-4 by T2h cells and immunoglobulin E by B cells; the reduction of cytokine IL-4 led to a decrease in Muc5AC expression. The specific mechanism of dexmedetomidine regulation of TSLP is not clear from this study.

\section{Conclusions}

1. Dexmedetomidine can attenuate airway hyperresponsiveness in the OVA asthma model.

Dexmedetomidine reduced the production of IL-4 and IgE through down-regulation of TLR4/NF-KB signalling pathway, thereby reducing the pulmonary inflammatory response and airway hyperresponsiveness in the OVA-induced asthma model.

\section{Declarations}

\section{Ethics approval and consent to participate}

Experimental animals were handled under a protocol approved by the Institutional Animal Care and Use Committee of Plastic Surgery Hospital, Chinese Academy of Medical Sciences and Peking Union Medical College

\section{Consent for publication}

All authors agree to publish

\section{Availability of data and materials}

The datasets used and/or analyzed during the current study are available from the corresponding author on reasonable request. 


\section{Competing interests}

The authors have declared that there is no conflict of interest.

\section{Funding}

None.

\section{Author's contributions}

Qianyu Wang,Dong Yang,: Conceived and designed the experiments. Xiyu DU,Juan Zhi and Qirui Duan: Performed the experimental and analyzed the data. Juan Zhi and Qirui duan:

Contributed reagents/materials/analysis tools. Qirui duan: Wrote the manuscript.

\section{Acknowledgements}

Not applicable.

\section{References}

1囚Reddel HK, Bateman ED, Becker A et al. A summary of the new GINA strategy: a roadmap to asthma control[J]. Eur Respir J. 2015;46(3):622-39. doi: 10.1183/13993003.00853-2015

2هGlobal Burden of Disease Study 2013 Collaborators. Global, regional, andnational incidence, prevalence, and years lived with disability for 301 acuteand chronic diseases and injuries in 188 countries, 1990-2013: a systematic analysis for the Global Burden of Disease Study 2013[J]. Lancet Lond. Engl. 2015,(386):743-800 . doi: 10.1016/S0140-6736(15)60692-4

$3 \llbracket$ Kewu Huang, Ting Yang , Jianying Xu et al. Prevalence, risk factors, and management of asthma in China: a national cross-sectional study[J].Lancet. 2019;394(10196):407-418; doi: 10.1016/S01406736(19)31147-X

$4 \llbracket$ Global Initiative for asthma, Global Strategy for Asthma Management and Prevention, Available from:, 2020 WWW.ginasthma.org;

5『Weifeng Tang , Ming Dong , Fangzhou Teng et al. TMT-based quantitative proteomics reveals suppression of SLC3A2 and ATP1A3 expression contributes to the inhibitory role of acupuncture on airway inflammation in an OVA-induced mouse asthma model[J].Biomed Pharmacother. 2021;134:111001;5. doi: 10.1016/j.biopha.2020.111001

6هE.D. Bateman, S.S. Hurd, P.J. Barnes et al. Global strategy for asthma management and prevention: GINA executive summary[J].Eur Respir J 2008;31:143-178; doi: 10.1183/09031936.00138707 
7 Chun-Hua Zhu , Jian Yu , Ben-Qing Wang et al. Dexmedetomidine reduces ventilator-induced lung injury via ERK1/2 pathway activation[J].Mol Med Rep.2020;22(6):5378-5384. doi: 10.3892/mmr.2020.11612

8\Jia Shi , Tianxi Yu , Kai Song et al. Dexmedetomidine ameliorates endotoxin-induced acute lung injury in vivo and in vitro by preserving mitochondrial dynamic equilibrium through the HIF-1a/HO-1 signaling pathway[J]. Redox Biol.2021;41:101954. doi: 10.1016/j.redox.2021.101954

9মKoichi Yuki.The immunomodulatory mechanism of dexmedetomidine[J].Int Immunopharmacol. 2021;97:107709. doi: 10.1016/j.intimp.2021.107709

10『Kun Wang , Mengge Wu, Jian Xu et al. Effects of dexmedetomidine on perioperative stress, inflammation, and immune function: systematic review and meta-analysis[J].Br J Anaesth 2019;123 (6) :777-794. doi: 10.1016/j.bja.2019.07.027.

11هHamida Hammad, Bart N Lambrecht.The basic immunology of asthma[J].Cell 2021;184(6): 14691485. doi: 10.1016/j.cell.2021.02.016.

12『Rong Li , leng K Lai, Jonathan Z Pan et al. Dexmedetomidine Exerts an Anti-inflammatory Effect via alpha2 Adrenoceptors to Prevent Lipopolysaccharide-induced Cognitive Decline in Mice[J]. Anesthesiology 2020;133 (2):393-407.doi: 10.1097/ALN.0000000000003390

13『Martijn L Manson, Jesper Säfholm, Anna James et al. IL-13 and IL-4, but not IL-5 nor IL-17A, induce hyperresponsiveness in isolated human small airways[J]. J Allergy Clin Immunol. 2020;145(3):808-817. doi: 10.1016/j.jaci.2019.10.037

14囚Bart N Lambrecht, Hamida Hammad, John V Fahy. The Cytokines of Asthma[J]. Immunity. 2019;50(4):975-991. doi: 10.1016/j.immuni.2019.03.018.

15 Erika von Mutius, Hermelijn H Smits. Primary prevention of asthma: from risk and protective factors to targeted strategies for prevention[J].Lancet. 2020;396(10254):854-866. doi: 10.1016/S01406736(20)31861-4.

16llkka S Junttil.Tuning the Cytokine Responses: An Update on Interleukin (IL)-4 and IL-13 Receptor Complexes[J].Front Immunol. 2018 Jun 7;9:888. doi: 10.3389/fimmu.2018.00888.

17囚Rongjun Wan, Juan Jiang, Chengping Hu et al.Neutrophil extracellular traps amplify neutrophil recruitment and inflammation in neutrophilic asthma by stimulating the airway epithelial cells to activate the TLR4/ NF-KB pathway and secrete chemokines[J].Aging (Albany NY). 2020;12(17):16820-16836. doi: 10.18632/aging.103479.

18凹Jin Shang, Weihua Liu, Chunyan Yin, Haiping Chu, Meizhen Zhang.Cucurbitacin E ameliorates lipopolysaccharide-evoked injury, inflammation and MUC5AC expression in bronchial epithelial cells by restraining the HMGB1-TLR4-NF-KB signaling[J].Mol Immunol. 2019;114:571-577. doi:

10.1016/j.molimm.2019.09.008.

Page 14/24 
19هM Yokota , T Tamachi , Y Yokoyama et al. IkBNS induces Muc5ac expression in epithelial cells and causes airway hyper-responsiveness in murine asthma models[J].Allergy. 2017;72(7): 1043-1053. doi: 10.1111/all.13079

20खLi-Jen Lin, Hui Ying Huang. DFSG, a novel herbal cocktail with anti-asthma activity, suppressed MUC5AC in A549 cells and alleviated allergic airway hypersensitivity and inflammatory cell infiltration in a chronic asthma mouse model[J].Biomed Pharmacother. 2020;121:109584. doi: 10.1016/j.biopha.2019.109584.

21区A William Tank, Dona Lee Wong. Peripheral and central effects of circulating catecholamines[J].Compr Physiol. 2015;5(1):1-15. doi: 10.1002/cphy.c140007.

22هQiying Shen, Guanghong Xu, Jing Liu, Lixia Wang et al. Dexmedetomidine alleviates non-ventilation associated lung injury via modulating immunology phenotypes of macrophages[J]. Life Sci. 2020;259:118249. doi: 10.1016/j.lfs.2020.118249.

23\X-H Du, S-S Li, G-S Xiong et al.Therapeutic efficacy of dexmedetomidine on chronic obstructive pulmonary disease via downregulating IncRNA PACER[J].Eur Rev Med Pharmacol Sci. 2020;24(24):12963-12970. doi: 10.26355/eurrev_202012_24200.

24囚Trevor P Creamer. Calcineurin[J].Cell Commun Signal. 2020;18(1):137.doi: 10.1186/s12964-02000636-4.

25『Morena Zusso, Valentina Lunardi, Davide Franceschin et al. Ciprofloxacin and levofloxacin attenuate microglia inflammatory response via TLR4/NF-KB pathway[J].J Neuroinflammation. 2019;16(1):148. doi: 10.1186/s12974-019-1538-9.

26खPegah Dejban, Nasrin Nikravangolsefid, Mohsen Chamanara, Ahmadreza Dehpour, Amir Rashidian. The role of medicinal products in the treatment of inflammatory bowel diseases (IBD) through inhibition of TLR4/NF-kappaB pathway[J].Phytother Res. 2021;35(2):835-845. doi: 10.1002/ptr.6866.

27囚Jing Liu, Xinhua Huang, Siping Hu, Huanzhong He, Zhipeng Meng et al. Dexmedetomidine attenuates lipopolysaccharide induced acute lung injury in rats by inhibition of caveolin-1 downstream signaling[J].Biomed Pharmacother. 2019;118:109314. doi: 10.1016/j.biopha.2019.109314.

28囚Eugene Kim, Hyun-Chang Kim, Seungmi Lee et al. Dexmedetomidine confers neur-op rotection against transient global cerebral ischemia/reperfusion injury in rats by inhibiting inflammation through inactivation of the TLR-4/NF-kB pathway[J].Neurosci Lett. 2017;649: 20-27. doi: 10.1016/j.neulet.2017.04.011.

29هLixia Wang, Muzi Wang, Shuai Li et al. Nebulized lidocaine ameliorates allergic airway inflammation via downregulation of TLR2[J].Mol Immunol. 2018;97:94-100. doi: 10.1016/j.molimm.2018.03.010. 
30凶Wei Cheng, Kun Yan, Yanni Chen et al. ABCA1 inhibits PDGF-induced proliferation and migration of rat airway smooth muscle cell through blocking TLR2/NF-KB/NFATc1 signaling

31هAlla Zamyatina, Holger Heine. Lipopolysaccharide Recognition in the Crossroads of TLR4 and Caspase-4/11 Mediated Inflammatory Pathways[J].Front Immunol. 2020;11:585146. doi: 10.3389/fimmu.2020.585146.

32هYoon-Young Sung, Seung-Hyung Kim, Heung Joo Yuk et al.Siraitia grosvenorii residual extract attenuates ovalbumin-induced lung inflammation by down-regulating IL-4, IL-5, IL-13, IL-17, and MUC5AC expression in mice.Phytomedicine. 2019;61:152835. doi: 10.1016/j.phymed.2019.152835.

33囚Sang-Hoon Lee, Seok-Rae Park. Toll-like Receptor 1/2 Agonist Pam3CSK4 Suppresses Lipopolysaccharide-driven IgG1 Production while Enhancing IgG2a Production by B Cells.Immune Netw. 2018;18(1):e10. doi: 10.4110/in.2018.18.e10.

34囚D S Ferreira, R Annoni, L F F Silva et al. Toll-like receptors 2, 3 and 4 and thymic stromal lymphopoietin expression in fatal asthma[J]. Clin Exp Allergy. 2012;42(10):1459-71. doi: 10.1111/j.13652222.2012.04047.x.

35खYoungwoo Choi, Young-Min Kim , Hee-Ra Lee et al. Eosinophil extracellular traps activate type 2 innate lymphoid cells through stimulating airway epithelium in severe asthma[J].Allergy. 2020;75(1):95-103. doi: 10.1111/all.13997

\section{Figures}




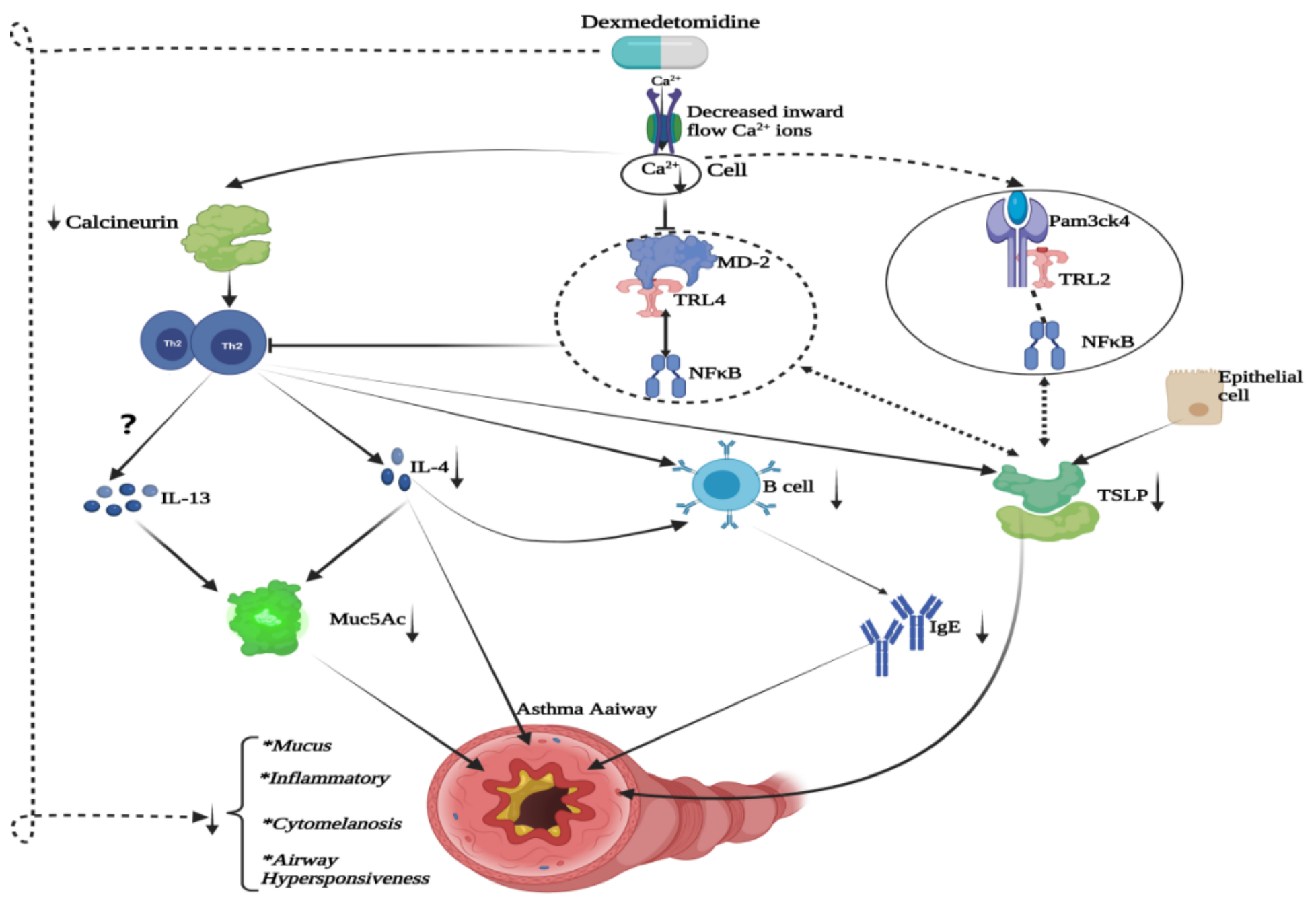

\section{Figure 1}

The present study suggests $\llbracket$ Dexmedetomidine attenuates inflammatory cytokines and airway hyperresponsiveness in models of asthma, and there are possible mechanisms by which. 1 . dexmedetomidine downregulates the TRL4/NFKB signalling pathway by inhibiting Ca2+ inward flow; 2 . down-regulation of TRL4/NFKB reduces stimulation of TH2 cells, thereby decreasing IL4 and IGE secretion and consequently Muc5AC protein expression; 3.The decrease in TSLP expression after dexmedetomidine application may be related to the downregulation of the TRL4/NFKB signalling pathway and possibly to attenuated epithelial cell stimulation; 4.The effect of dexmedetomidine on $\mathrm{TH} 2$ cells may have the involvement of calcium-regulated neurophosphatase. 


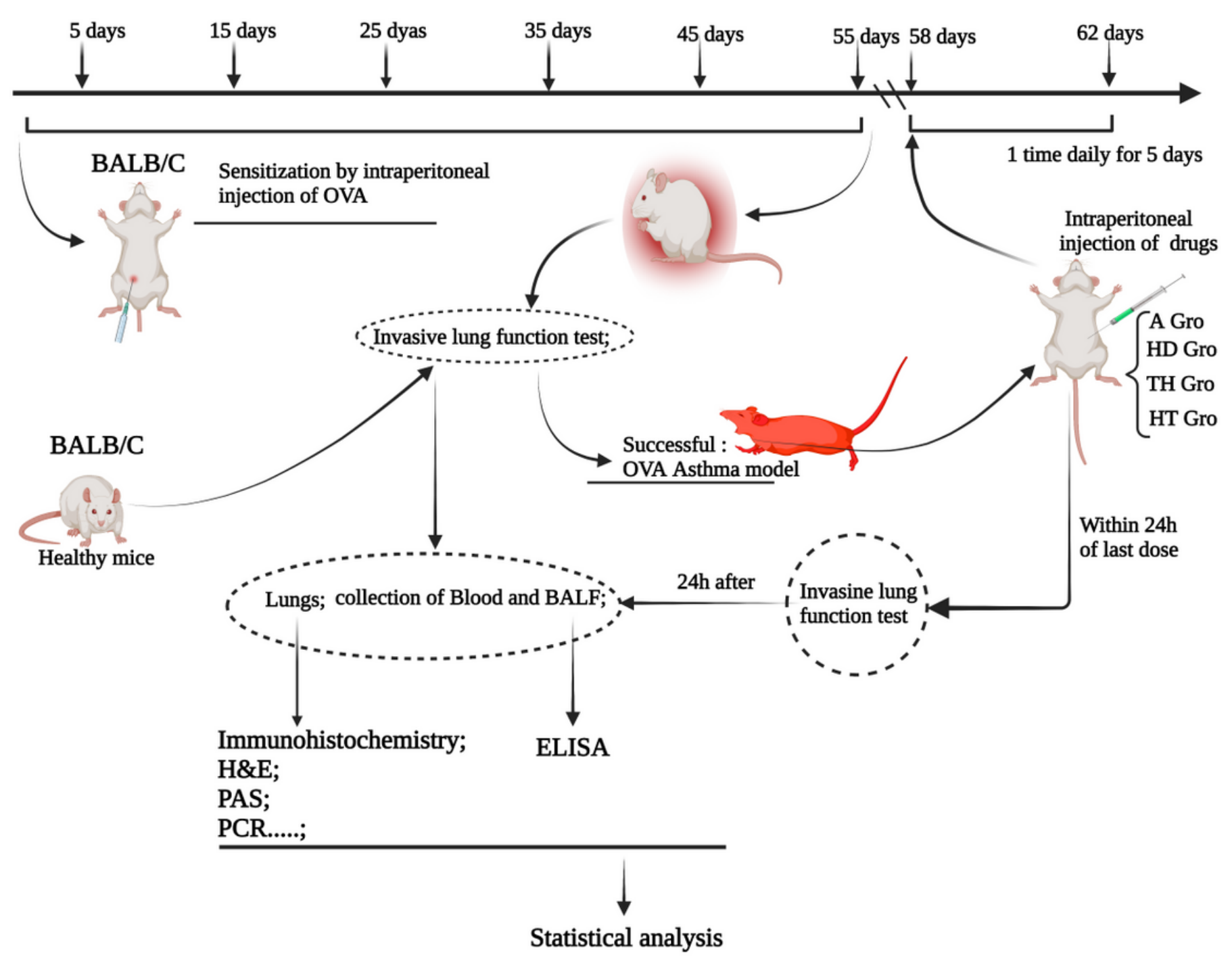

Figure 2

Experimental process diagram. 

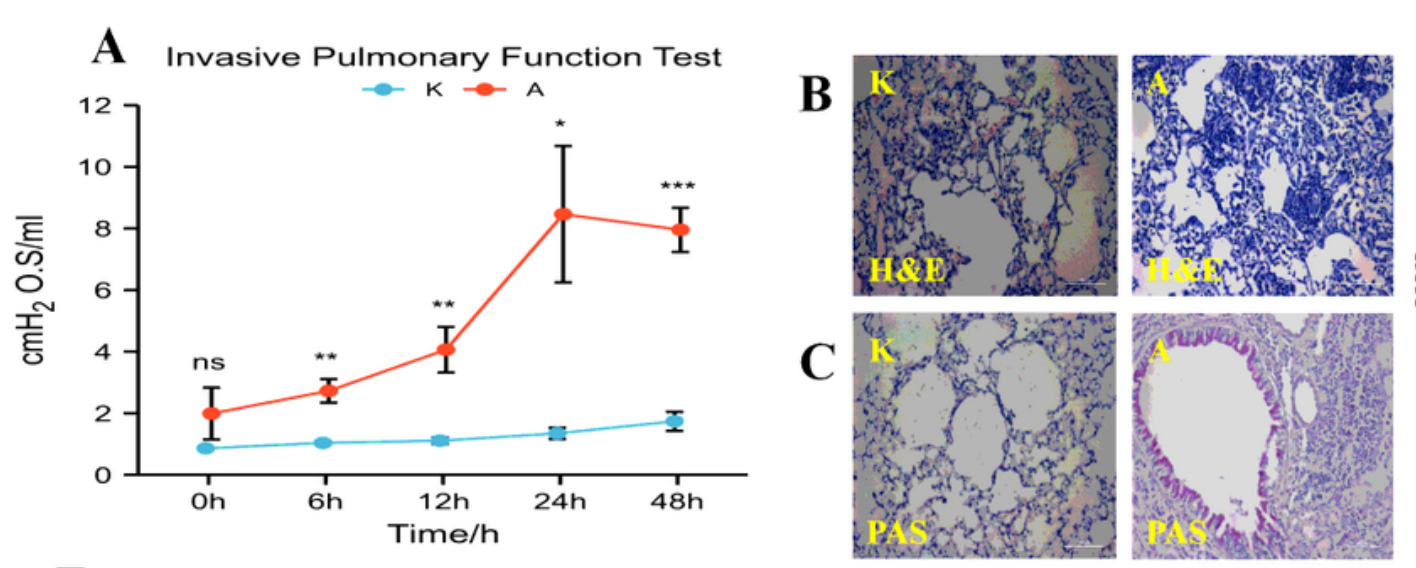

\section{D}

Percentage of areas after $\mathrm{HE}$ and PAS
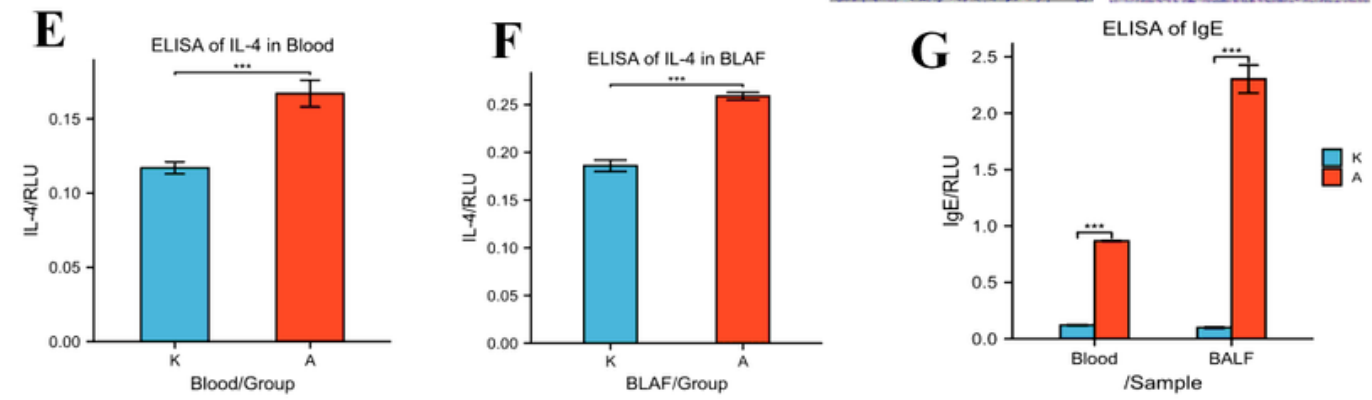

$\mathbf{H}$

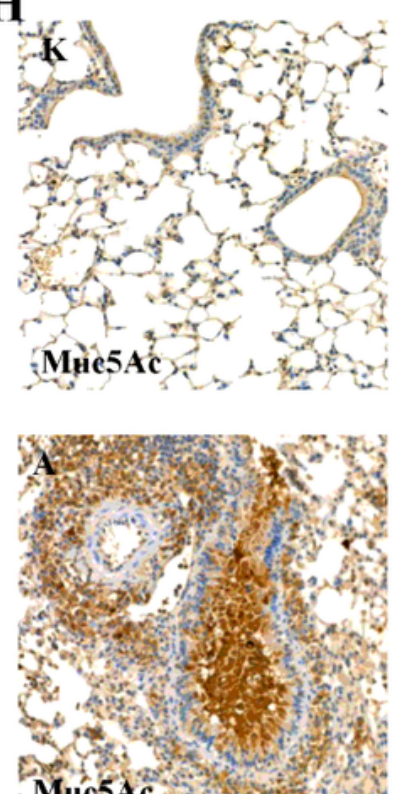

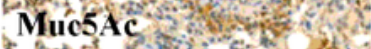
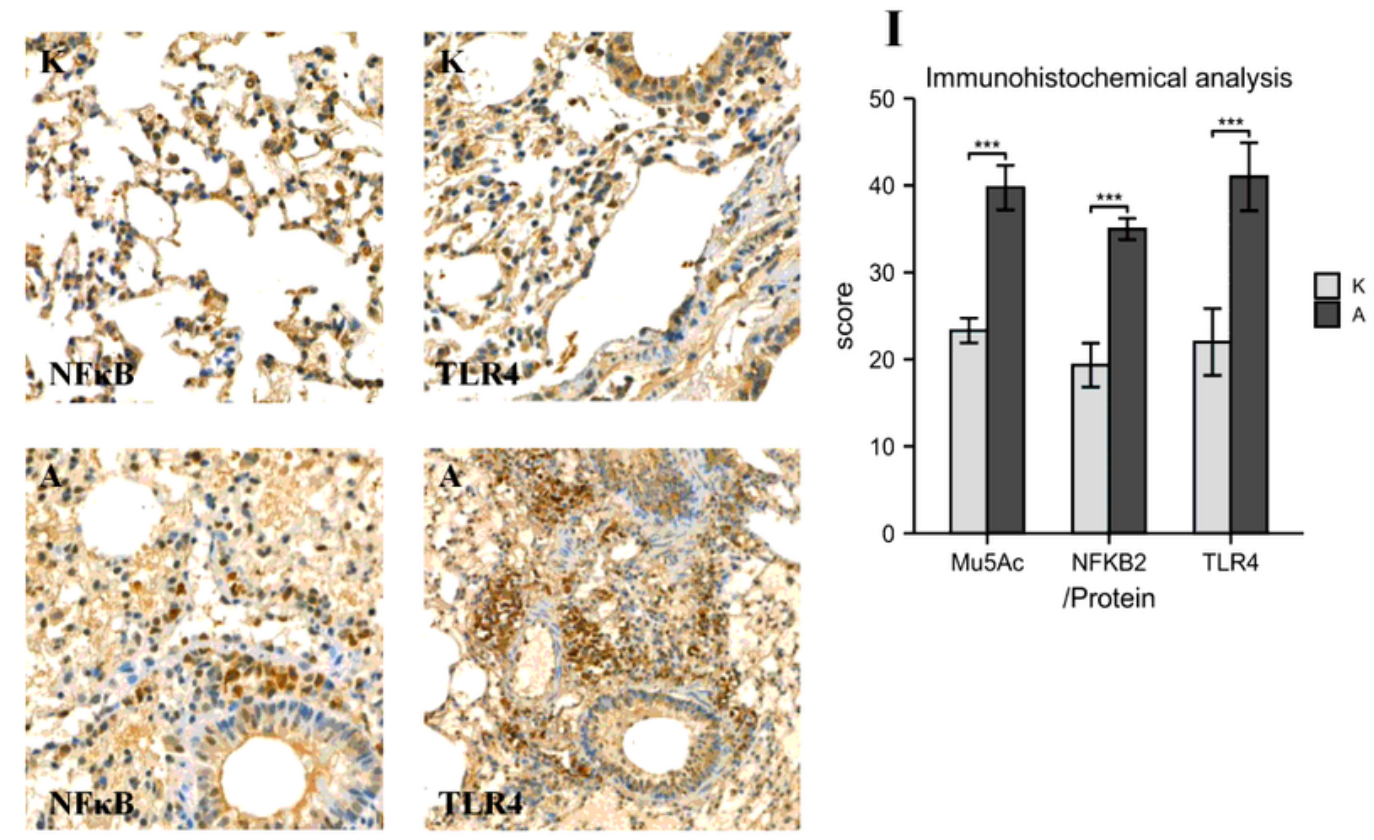

\section{Figure 3}

A shows that the airway resistance was significantly higher in the asthma group than in the blank group after passing the modelling, and all were statistically different after $6 \mathrm{~h}$; B, shows the HE staining results.C, shows PAS staining results. By $\mathrm{B} \square \mathrm{C} \square \mathrm{D}$, we know that after the asthma model, there was severe structural destruction of the lung, increased inflammatory infiltration, increased sputum, and cupped cell hyperplasia. Based on E $\mathrm{F} \square \mathrm{G}$, we know that IL-4 and IGE in both peripheral serum and BALF were 
increased in the asthma model. His the result of immunohistochemistry of lung tissue. According to $\mathrm{H}$ and I, Muc5AC, TRL4/NFKB were increased in lung tissues of the asthma model.

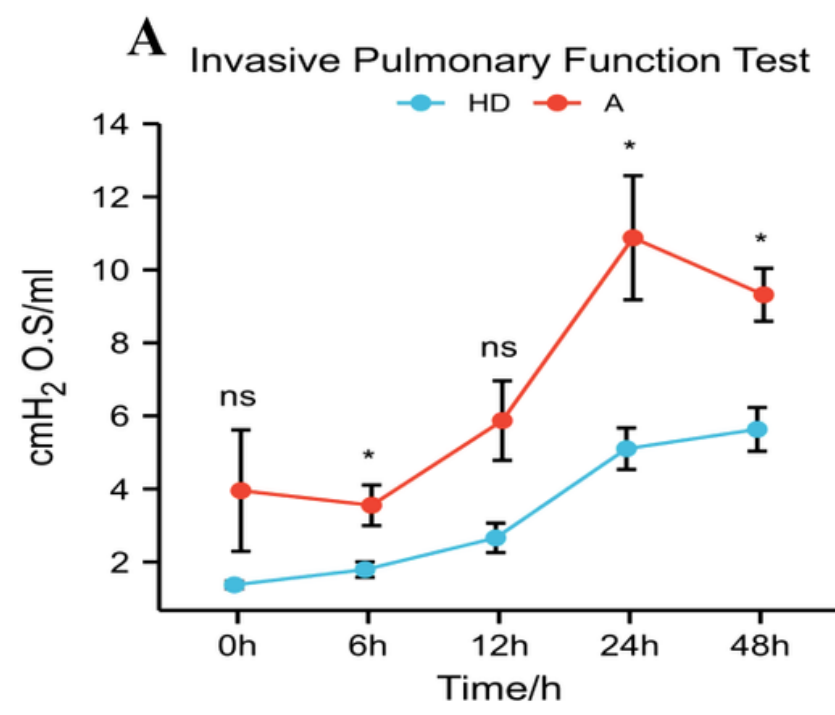

Pairwise Comparisons of Estimated Marginal Means

\begin{tabular}{cccc}
\hline $\begin{array}{c}\text { Horizontal } \\
\text { groups (time) }\end{array}$ & Group I & Group J & $\begin{array}{c}\text { Corrected } \boldsymbol{P} \text { - } \\
\text { value }\end{array}$ \\
\hline $0 \mathrm{~h}$ & HD & $\mathrm{A}$ & 0.196 \\
$6 \mathrm{~h}$ & HD & $\mathrm{A}$ & 0.041 \\
$12 \mathrm{~h}$ & HD & $\mathrm{A}$ & 0.050 \\
$24 \mathrm{~h}$ & HD & $\mathrm{A}$ & 0.032 \\
$48 \mathrm{~h}$ & HD & $\mathrm{A}$ & 0.017 \\
\hline
\end{tabular}

*The effect of two-by-two comparison of longitudinal subgroups (X1) on observed variables at different cross-sectional subgroups (time): in the $0 \mathrm{~h}$ subgroup, $\mathrm{A}$ was higher than the mean of $\mathrm{HD}$, the difference was not statistically significant ( $\mathrm{P}=0.196)$; in the $6 \mathrm{~h}$ subgroup, A was higher than the mean of $\mathrm{HD}$, the difference was statistically significant $(\mathrm{P}=0.041)$; in the $12 \mathrm{~h}$ subgroup, A was higher than the mean of HD The difference was not statistically significant $(\mathrm{P}=0.050)$; in the $24 \mathrm{~h}$ subgroup, A was higher than the mean level of $\mathrm{HD}$, and the difference was statistically significant $(\mathrm{P}=0.032)$; in the $48 \mathrm{~h}$ subgroup, $\mathrm{A}$ was higher than the mean level of $\mathrm{HD}$, and the difference was statistically significant $(\mathrm{P}=0.017)$

Figure 4

The graph shows that airway resistance decreased significantly at all time periods after dexmedetomidine treatment compared to the asthma group. However, interestingly no statistical difference was seen at the $12 \mathrm{~h}$ time point $(P=0.05)$ 
A
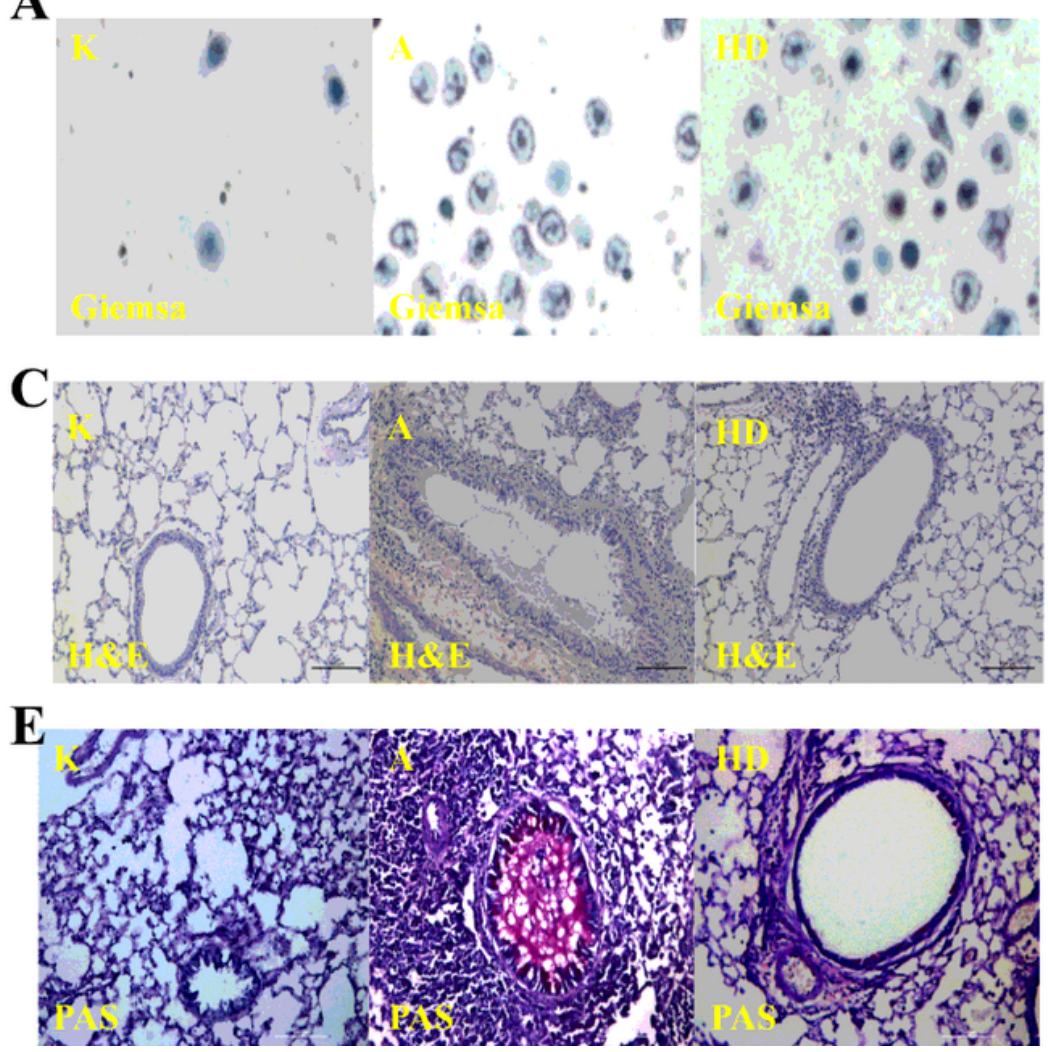

B
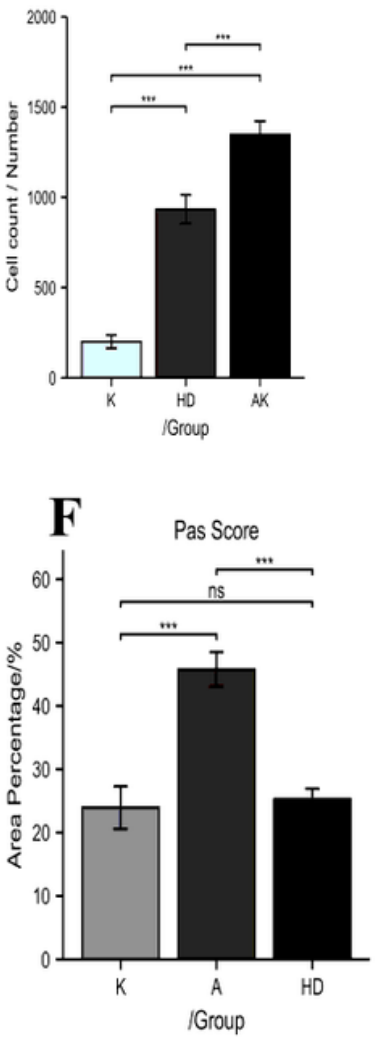

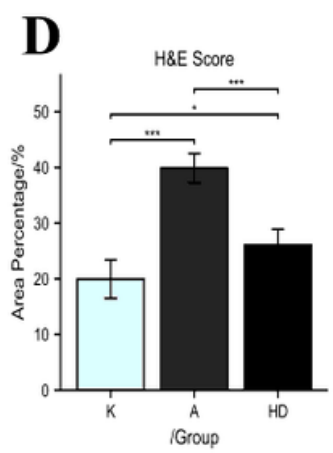

Figure 5

A shows that the total number of eosinophils and neutrophils was significantly reduced after dexmedetomidine treatment by Kimsa staining of a portion of BALFA fluid. B $\triangle \square \square$ shows retarded structural destruction of the lung, diminished cuprocyte chemotaxis and reduced mucus after dexmedetomidine treatment. 

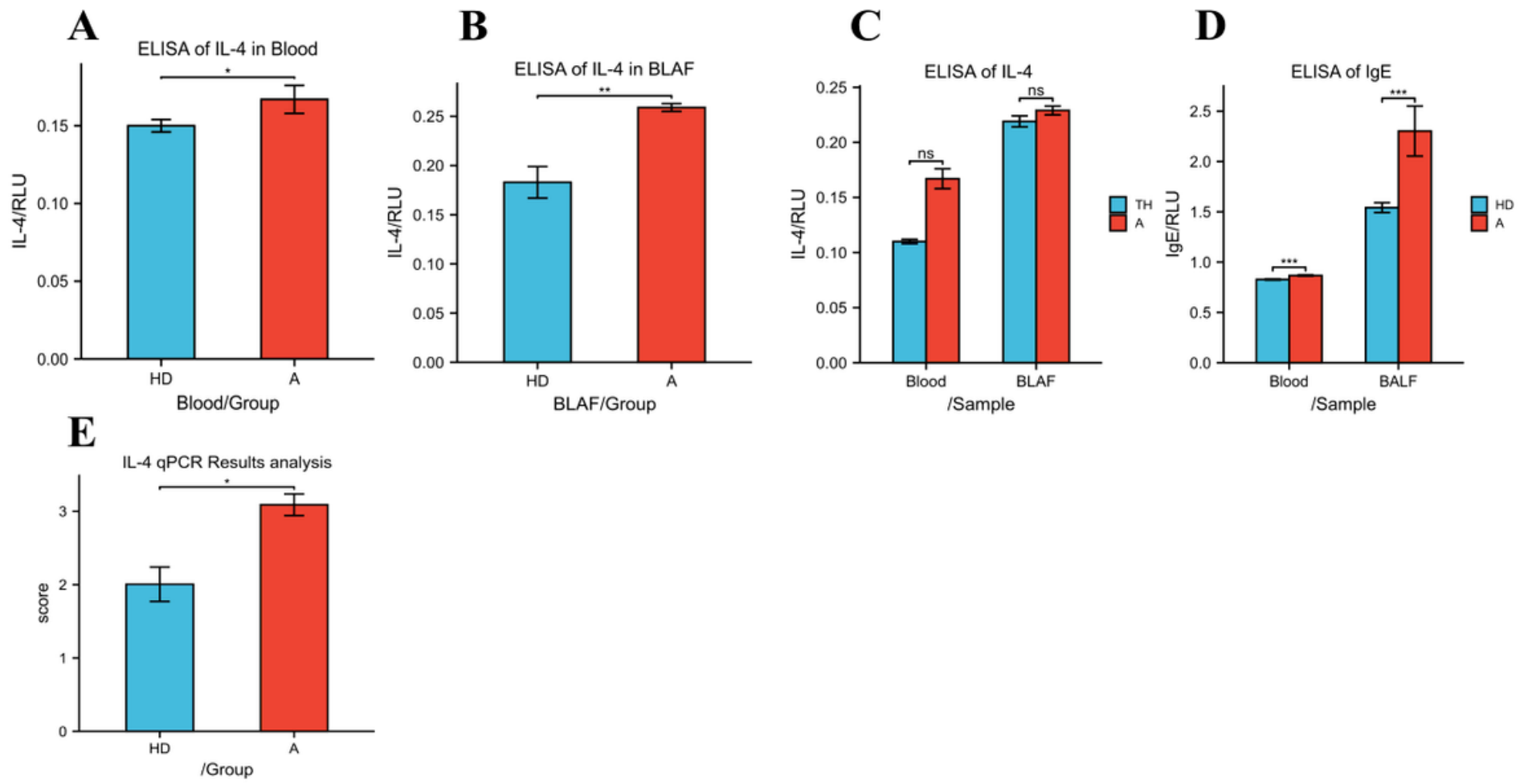

Figure 6

Graphs A, B and D show a decrease in IL-4 and ige in serum and BALF after dexmedetomidine treatment compared to the asthma group (group A). There was also a decrease in IL-4 in lung tissue as verified by pcr testing, as shown in E. Yohimbine was also used as a drug control group and was found to have no significant effect on IL-4 in serum and BALF, which is shown in C. 
A
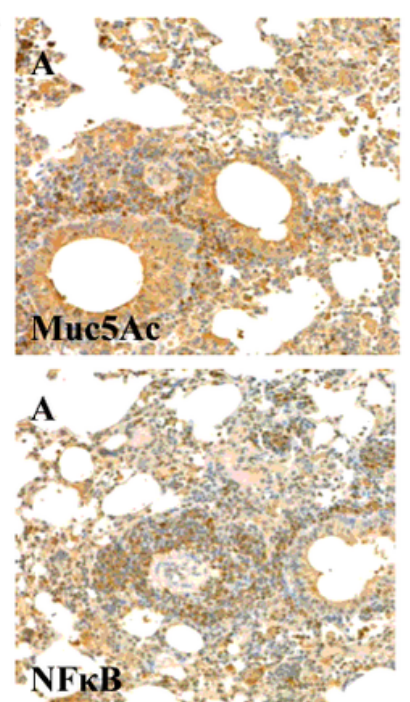

NFKB

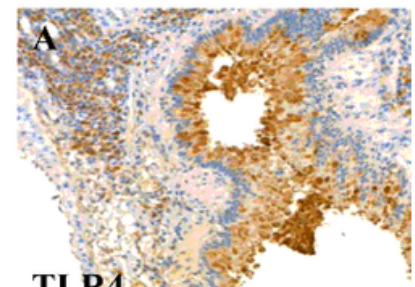

TLR4
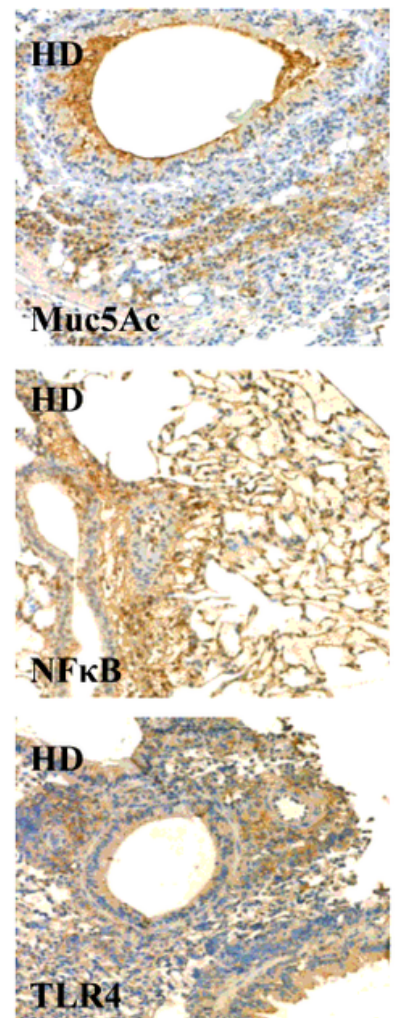
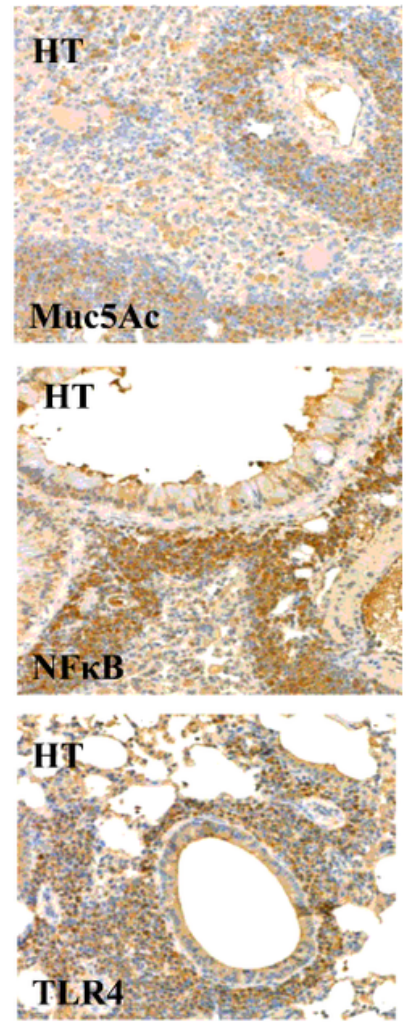

B

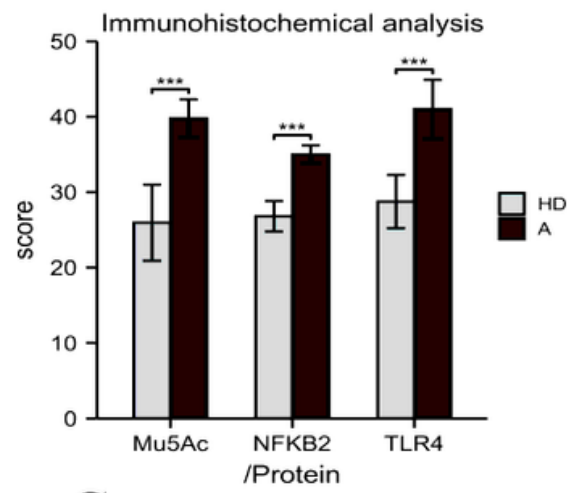

C

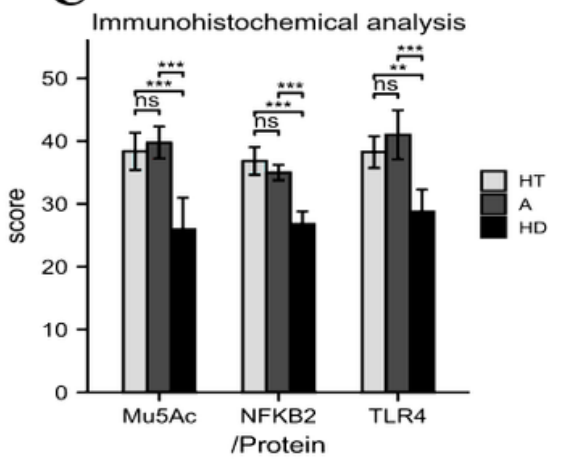

\section{Figure 7}

A shown, is the expression of Muc5AC, TRL4/NFKB in immunohistochemistry in the lung tissue of each group in response to group A, HD and HT. By B we can know that the expression of Muc5AC, TRL4/NFKB all decreased after dexmedetomidine treatment and this effect was affected by yohimbine. 


\section{A}

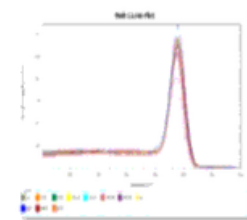

IL4 Melt Curve

Plot-A

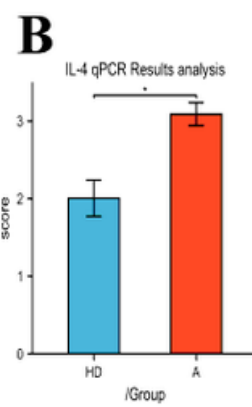

A

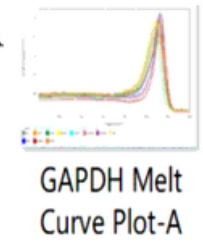

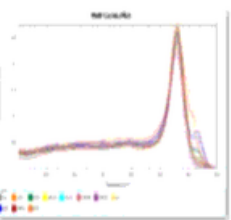

IL13 Melt Curve

Plot-A

C

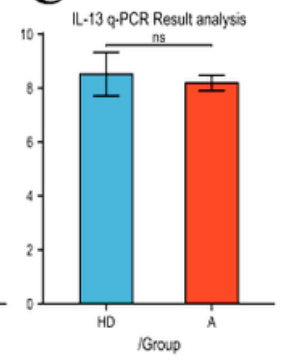

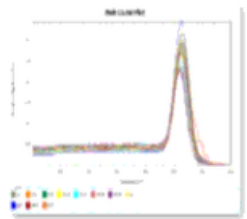

Muc5AC Melt

Curve Plot-A

D

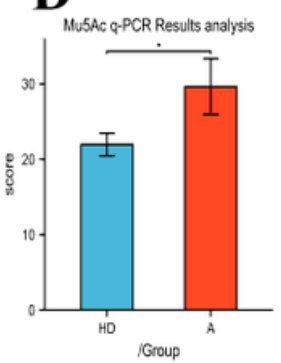

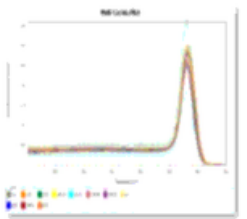

NF-kB Melt

Curve Plot-A

$\mathbf{E}_{\text {NFBB Q PCCR Result anylys }}$

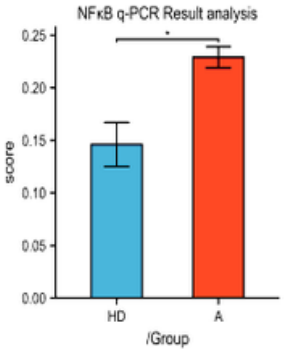

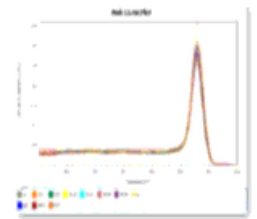

TLR2 Melt

Curve Plot-A

F

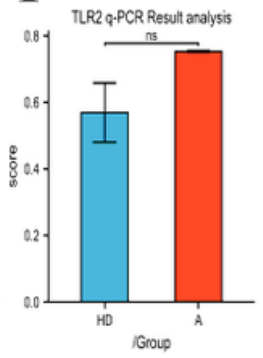

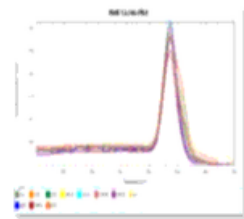

TLR4 Melt

Curve Plot-A

G

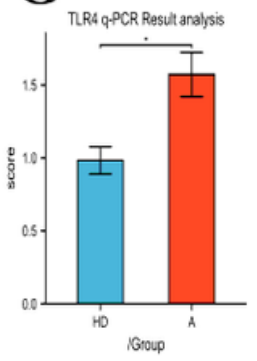

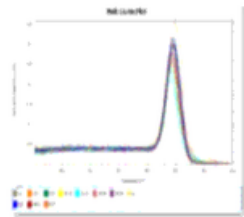

TSLP1 Melt

Curve Plot-A

H

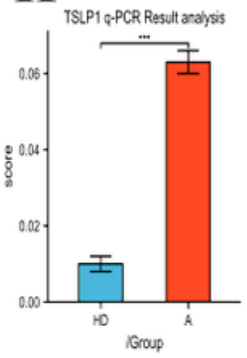

Figure 8

The PCR dissolution curves shown in graph A. B to H show the protein content of IL-4, IL-13, Muc5A, CTLR2, TLR4 and TSLP1 in lung tissue of the HD group compared to group A. The difference between IL4, Muc5A, TLR4 and TSLP1 is statistically significant. Among them, IL-4, Muc5A, TLR4 and TSLP1 were statistically different.

\section{Supplementary Files}

This is a list of supplementary files associated with this preprint. Click to download.

- Graphicalabstract.docx 